\title{
Channel Diversity needed for Vector Interference Alignment
}

Cheuk Ting Li and Ayfer Özgür

Department of Electrical Engineering, Stanford University, Stanford, CA 94305

\section{Background}

Interference is the central phenomenon severely limiting the performance of most wireless systems

$\checkmark$ Interference alignment has been recently proposed to mitigate interference

$\checkmark$ Design signals such that, upon arriving at unintended receivers, they overlap with each other and the resulting interference is perceived as much less than the sum of the individual interferences

$\checkmark$ Interference alignment achieves K/2 degrees of freedom

$\checkmark$ At high-SNR, each user can communicate as if it has half the resources of the channel for its exclusive use, independent of the number of users

$\checkmark$ However, the construction requires the channel diversity, i.e., the number of independent channel realizations (e.g. the time needed, or the bandwidth), to grow exponentially in the number of users

$\checkmark$ Our goal is to study the trade-off between DoF and channel diversity

\section{Channel Model}

Consider K-user fully-connected real-valued fast-fading interference channel

$\checkmark$ Each receiver wants to obtain a message from its corresponding transmitter, but the signal is superimposed by interferences from other transmitters

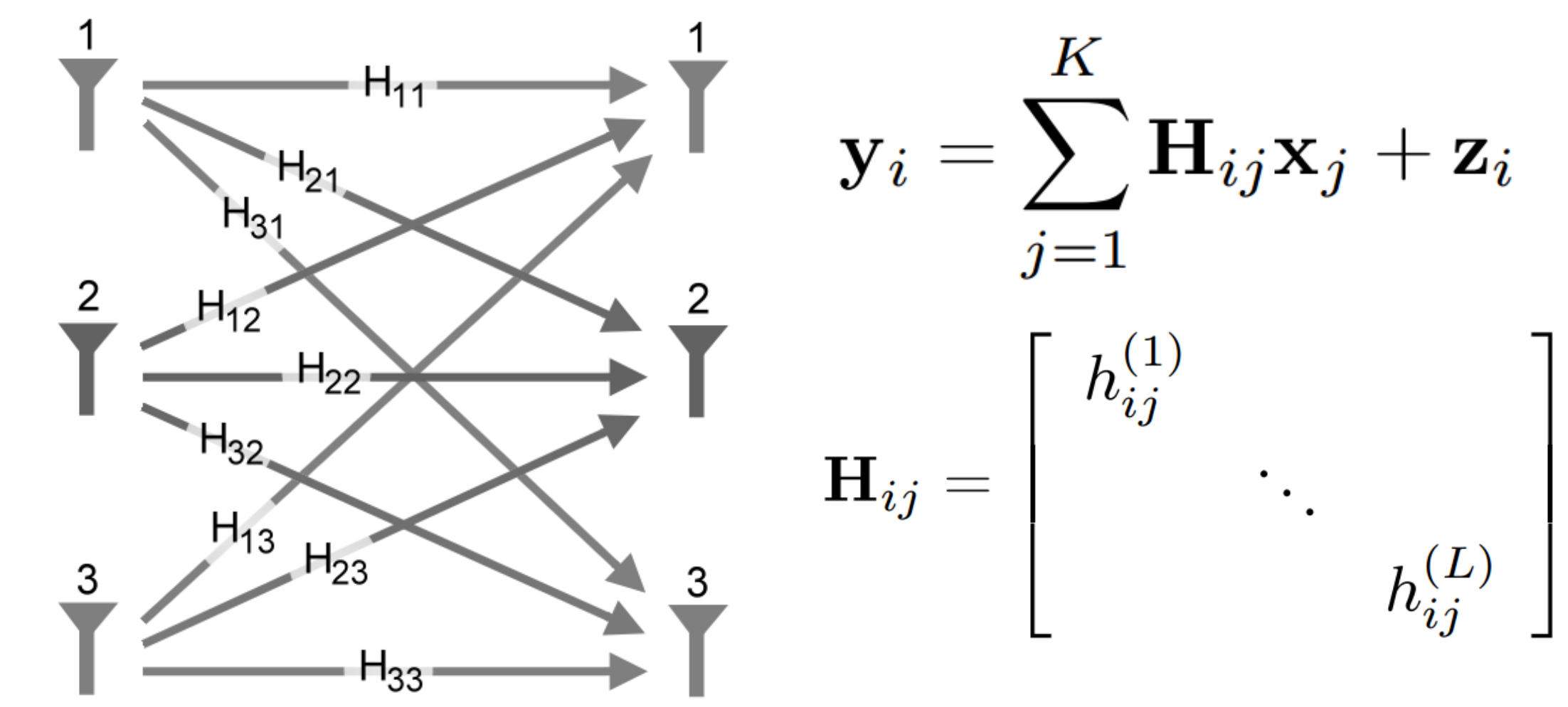

\section{Interference Alignment}

$\checkmark$ Vector space interference alignment strategies [1]:

Message vector $\hat{\mathbf{x}}_{i} \in \mathbb{R}^{D}$, apply linear precoding $\mathbf{x}_{i}=\mathbf{V}_{i} \hat{\mathbf{x}}_{i}$ to map $\hat{\mathbf{x}}_{i}$ to a subspace $V_{i}$, receiver decodes by zero forcing interference

$\checkmark$ Decoding is successful if signal subspace is disjoint from interference subspace

$$
\mathbf{H}_{i i} V_{i} \cap\left(\sum_{j \neq i} \mathbf{H}_{i j} V_{j}\right)=\{0\}
$$

$$
\text { DoF }=\underset{\left\{V_{i}\right\} \text { satisfies decoding condition } \forall i}{\max }
$$

\section{Trade-off between DoF and Diversity}

$\diamond$ For 3-user interference channel [2],

$$
\mathrm{DoF}=\frac{3}{2}\left(1-\frac{1}{4 L-2\lfloor L / 2\rfloor-1}\right)
$$

The gap between the DoF and $\mathrm{K} / 2$ decreases as $1 / \mathrm{L}$

$\checkmark$ We show that for $K \geq 4$ users,

$$
\operatorname{DoF} \leq \frac{K}{2}\left(1-\frac{1}{11 \sqrt{L}}\right)
$$

The gap between DoF and $K / 2$ decreases at most as $1 / \sqrt{L}$

$\checkmark$ Moreover, if $\mathrm{K}$ is larger than the order of $\sqrt{\log L}$, then the gap decreases at most as $1 / \sqrt[4]{L}$

\section{Alignment Width}

$\checkmark$ Define the alignment width of a subspace as

$$
\begin{aligned}
\Delta_{\mathbf{T}} V & =\operatorname{dim} V-\operatorname{dim}(V \cap \mathbf{T} V) \\
& =\operatorname{dim}(V+\mathbf{T} V)-\operatorname{dim} V
\end{aligned}
$$

$\checkmark$ The subspaces $V_{i}$ need to align to the transformations $\mathbf{T}_{i j k}=\mathbf{H}_{1 i}^{-1} \mathbf{H}_{1 k} \mathbf{H}_{j k}^{-1} \mathbf{H}_{j i}$

$\diamond$ If $\operatorname{DoF} \geq(1-\epsilon) K / 2$, then $\Delta_{\mathbf{T}_{i j k}} V_{i} \leq 2 \epsilon L$

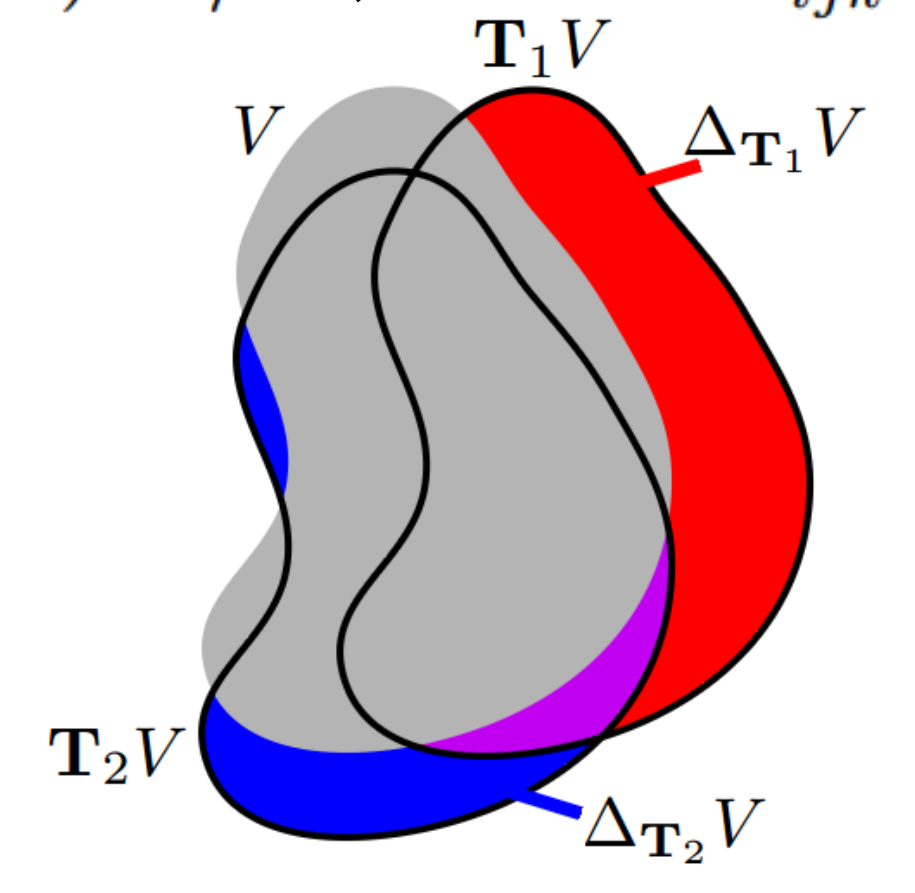

\section{Intuition of the Proof}

$\checkmark$ Compared to $V$, the dimension of $V \cap \mathbf{T}_{1} V$ is reduced by $\Delta_{\mathbf{T}_{1}} V$

$\diamond$ As long as $n_{1} \Delta_{\mathbf{T}_{1}} V<D, V \cap \cdots \cap \mathbf{T}_{1}^{n_{1}} V \neq\{0\}$

$\diamond$ We can find $\mathbf{v}$ such that $\operatorname{span}\left\{\mathbf{v}, \mathbf{T}_{1} \mathbf{v}, \ldots, \mathbf{T}_{1}^{n_{1}} \mathbf{v}\right\} \subseteq V$

$\diamond$ As long as $n_{2} \Delta_{\mathbf{T}_{2}} V<L-D, V+\cdots+\mathbf{T}_{2}^{n_{2}} V \neq \mathbb{R}^{L}$

$\diamond$ Hence $\operatorname{span}\left\{\mathbf{T}_{1}^{a} \mathbf{T}_{2}^{b} \mathbf{v}\right\}_{a=0, \ldots, n_{1}, b=0, \ldots, n_{2}} \neq \mathbb{R}^{L}, n_{1} n_{2} \leq L$
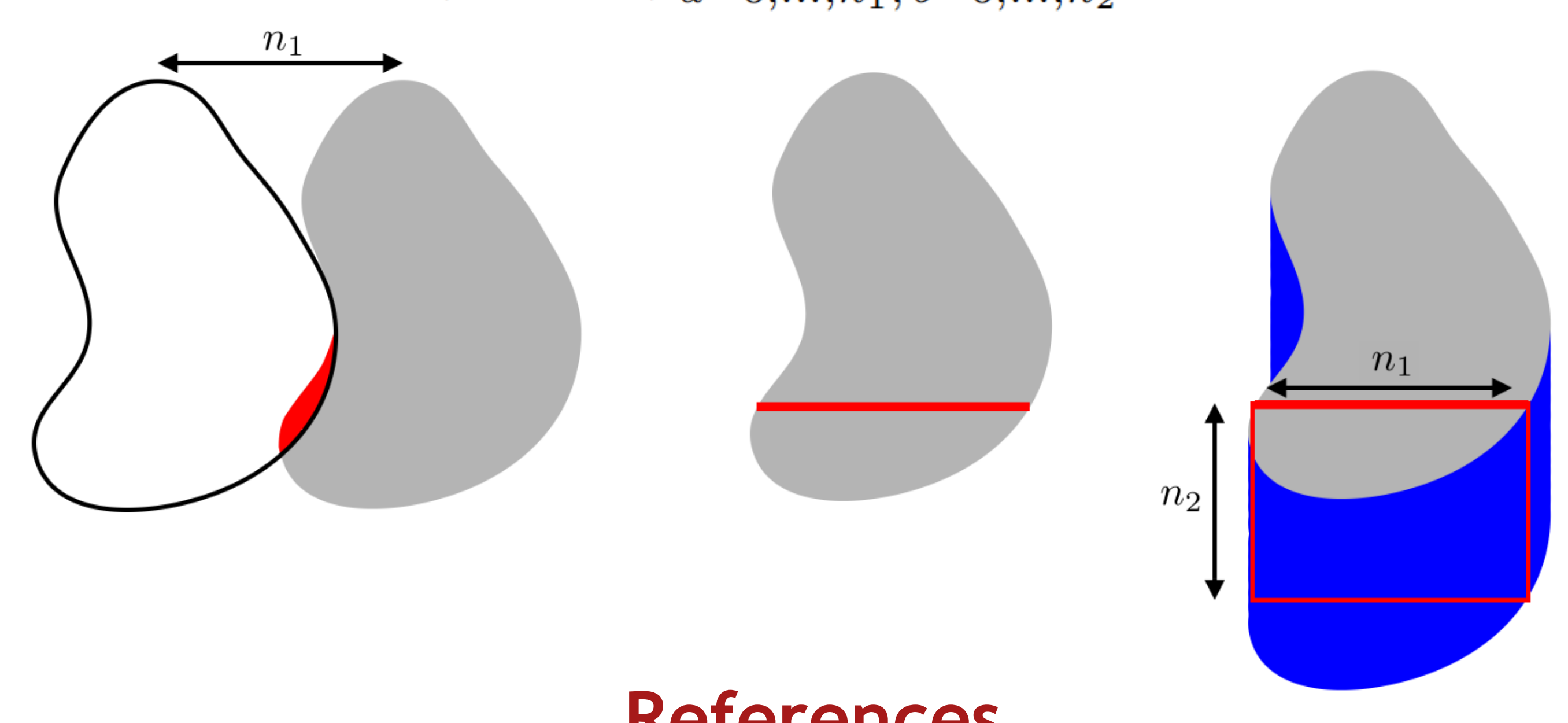

References

[1] V. Cadambe and S. A. Jafar, “Interference alignment and degrees of freedom of the K-user interference channel," IEEE
Trans. Inf. Theory, vol. 54, no. 8, pp. 3425-3441, Aug. 2008.

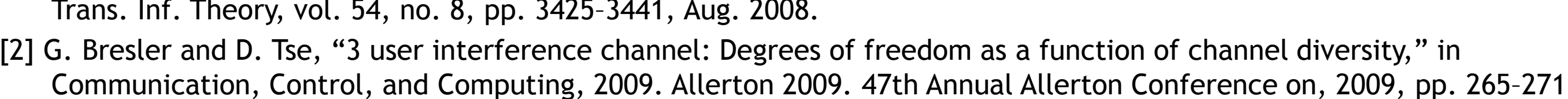

